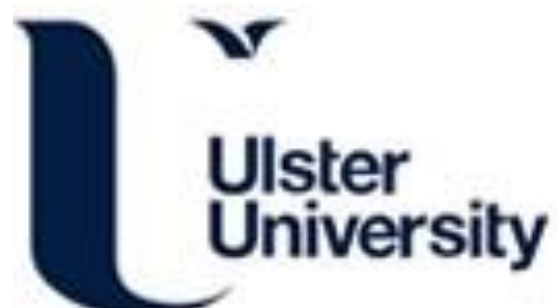

\section{Detection of fast and slow hand movements from motor imagery EEG signals}

Bhattacharyya, S., Hossain, M. A., Konar, A., Tibarewala, D. N., \& Ramadoss, J. (2014). Detection of fast and slow hand movements from motor imagery EEG signals. In Advanced Computing, Networking and Informatics Proceedings of the Second International Conference on Advanced Computing, Networking and Informatics, ICACNI 2014 (VOL 1 ed., pp. 645-652). (Smart Innovation, Systems and Technologies; Vol. 27, No. VOL 1). Springer Science and Business Media Deutschland GmbH. https://doi.org/10.1007/978-3-319-07353-8_74

Link to publication record in Ulster University Research Portal

Published in:

Advanced Computing, Networking and Informatics - Proceedings of the Second International Conference on Advanced Computing, Networking and Informatics, ICACNI 2014

Publication Status:

Published (in print/issue): 01/01/2014

DOI:

10.1007/978-3-319-07353-8_74

\section{Document Version}

Author Accepted version

\section{General rights}

Copyright for the publications made accessible via Ulster University's Research Portal is retained by the author(s) and / or other copyright owners and it is a condition of accessing these publications that users recognise and abide by the legal requirements associated with these rights.

\section{Take down policy}

The Research Portal is Ulster University's institutional repository that provides access to Ulster's research outputs. Every effort has been made to ensure that content in the Research Portal does not infringe any person's rights, or applicable UK laws. If you discover content in the Research Portal that you believe breaches copyright or violates any law, please contact pure-support@ulster.ac.uk. 


\title{
Detection of Fast and Slow Hand Movements from Motor Imagery EEG Signals
}

\author{
Saugat Bhattacharyya ${ }^{1}$, Munshi Asif Hossain ${ }^{1}$, Amit Konar ${ }^{1}$, D.N. \\ Tibarewala $^{2}$, and R. Janarthanan ${ }^{3}$ \\ ${ }^{1}$ Dept. of Electronics and Telecommunication Engineering, ${ }^{2}$ School of Bioscience \\ and Engineering, Department of Computer Science, \\ ${ }^{1,2}$ Jadavpur University, Kolkata, India ${ }^{3}$ TJS Engineering College, Chennai, India \\ \{saugatbhattacharyya@gmail.com, asif_ju@live.com, konaramit@yahoo.co.in, \\ biomed.ju@gmail.com, srmjana_73@yahoo.com,
}

\begin{abstract}
Classification of Electroencephalography (EEG) signal is an open area of re-search in Brain-computer interfacing (BCI). The classifiers detect the different mental states generated by a subject to control an external prosthesis. In this study, we aim to differentiate fast and slow execution of left or right hand move-ment using EEG signals. To detect the different mental states pertaining to motor movements, we aim to identify the event related desynchronization/ synchronization (ERD/ERS) waveform from the incoming EEG signals. For this purpose, we have used Welch based power spectral density estimates to create the feature vector and tested it on multiple support vector machines, Nave Bayesian, Linear Discriminant Analysis and k-Nearest Neighbor classifiers. The classification accuracies produced by each of the classifiers are more than $75 \%$ with nä̈ve Bayesian yielding the best result of $97.1 \%$.
\end{abstract}

Keywords: Motor imagery, Brain-computer interfacing, Event related desynchronization/ synchronization, Electroencephalography, Pattern classifiers

\section{Introduction}

Brain-computer interfacing (BCI) is a field of study which aims to provide a communication channel between the mental commands generated from the brain of a user and an external device (say, a robot) without any muscular intervention [1]. Earlier studies in BCI aimed at providing rehabilitation to physically challenged patients suffering from Amytrophic Lateral Sclerosis (ALS), cerebral palsy, stroke, paralysis and amputee [2]. But recent advances in BCI has opened newer fields of application in communication and control, gaming, robotics and military [3-5].

Electroencephalography (EEG) is the most frequently used form of brain measure because it is non-invasive, portable, easy to use, widely available, and has a good temporal resolution [6]. EEG is a non-linear, non-stationary, nonGaussian signal [1] and for this reason information relating to the mental states 
of the user cannot be ascertained directly from the raw data. Thus, to extract the relevant features and classify the mental states of the user, an EEG-BCI module is made of the following components: i) Pre-processing, where the incoming signals from the EEG amplifier are filtered to the required frequency bands, ii) Feature Extraction, where the characteristic information from the filtered EEG signals are extracted using signal processing algorithms, and iii) Classification, to decode between the different mental states from the EEG signal [7]. Existing literatures in BCI have widely used time-, frequency-, time-frequency-, and nonlinear domain as feature extractors $[1,3,5,6,8]$ and algorithms like Support Vector Machine (SVM), Linear Discriminant Analysis (LDA), Nave Bayesian and Neural Networks for classification [9].

Different mental states leads to generation of diverse brain signal modalities. A few well known ones are steady-state visually evoked potential (SSVEP), slow cortical potential (SCP), P300, event related desynchronization/synchronization (ERD/ERS) and error related potential (ErRP)[2]. The selection of brain signals (or signal modality), is an important issue in EEG-BCI analysis and it depends on the cognitive task performed by the subject. ERD/ERS originates during motor planning, imagination or execution (also referred to as motor imagery signals)[10] and thus, this signal finds relevance in our study. ERD/S waveforms are pre-dominantly obtained from the alpha (8-12 Hz) and central beta (16-24 $\mathrm{Hz})$ rhythm[2].

Existing literatures [11-14] on motor imagery EEG signal classification have extensively classified between left and right motor imagery signals. In this paper, we aim to classify the speed of execution of the motor imagery tasks given to the subjects. For this purpose, we have designed a two-level hierarchical classification strategy, where the first level classifies between the left and right hand movement and the second level differentiates between fast and slow movement of the respective limb (as decided in the first level). Here, we have employed Welch based Power Spectral Density estimates in the alpha and central-beta band as features and linear discriminant analysis, k-nearest neighbors, nä̈ve Bayesian and Support Vector Machines as classifiers. The results thus obtained, suggests that our proposed classification strategy is successful in classifying the mental states of fast and slow movement for both the left and right hand.

The rest of the paper is organized as follows: Section 2 describes the details of the experiments along with the data processing and classification strategies implemented in this study, the results are discussed in Section 3 followed by the concluding remark in Section 4.

\section{Experiments and methods}

In this study, we have separated the fast and slow execution of the left and right hand movement according to the instructions given to the subject. The complete scheme for this study is given in Fig. 1. Following the data acquisition of EEG signals according to different mental states, the first step involves the filtering of the raw signal. In the second step, the features are extracted using Power 
Spectral Density leading to the formation of the feature vectors. The feature vectors are used as inputs to the classifiers to determine the mental state of the user. Nine right-handed subjects ( 4 male and 5 female) have participated in this study over 3 separate sessions organized in 3 different days.

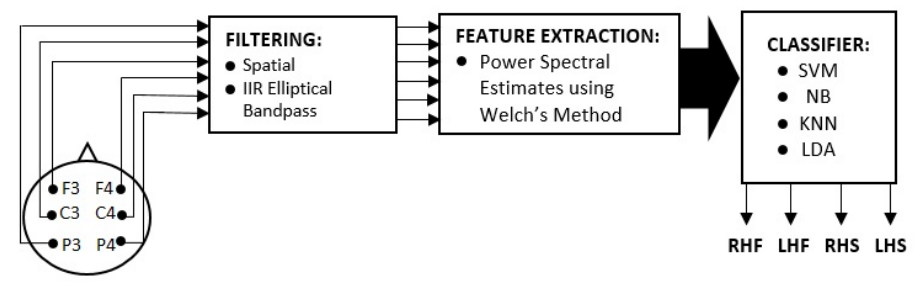

Fig. 1. A block diagram representation of our overall scheme.

\subsection{Visual Cue}

The subjects perform the motor execution tasks based on the instructions given on a visual cue. Each session consists of 100 trials (25 each for right-hand fast move-ment (RHF), left-hand fast movement (LHF), right-hand slow movement (RHS) and left-hand slow movement (LHS)). The timing scheme for each session (as shown in Fig. 2) are as follows: In the first second, a fixation cross ' + ' is displayed on screen which is an indication to the subject to get ready to perform the task. In the next screen, instruction is given to the subject on the speed of execution, i.e., slow and fast movement. This screen lasts for 1 second following which left or right arrow is displayed according to which the subject moves the respective limb either slowly or briskly (based on the instruction in the previous screen) for 3 seconds. At the end of each trial, a blank screen of 2 seconds is displayed during which the subject can relax.

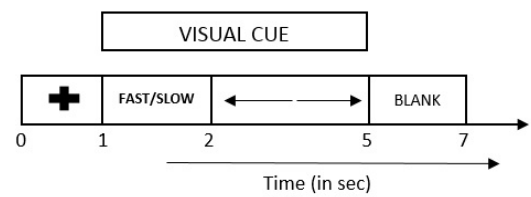

Fig. 2. The timing scheme diagram of a visual cue 


\section{$2.2 \quad$ Experimental setup}

In this experiment, a NeuroWin (manufactured by NASAN) EEG machine with 19 electrodes ( Fp1, Fp2, F8, F4, Fz, F3, F7, T4, C4, Cz, C3, T5, T6, P4, Pz, $\mathrm{P} 3, \mathrm{~T} 7, \mathrm{O} 2, \mathrm{O} 1)$ is used to record the mental states of the subjects. The left ear is used as a reference and $\mathrm{FPz}$ location is grounded. The EEG is recorded using gold plated electrodes and the impedances are kept below $5 \mathrm{k} \Omega$. The EEG signals are amplified, sampled at $250 \mathrm{~Hz}$, and band-pass filtered between 0.5 and $35 \mathrm{~Hz}$.

\section{$2.3 \quad$ Pre-processing}

Motor imagery signals originates from the primary motor cortex, supplementary motor area and pre-motor cortex from the brain [2]. Thus, locations in between the frontal and parietal lobe contains the maximum information on motor related tasks. For this purpose, we have analyzed the signals acquired from F3, F4, C3, C4, P3 and P4 electrode locations in this study. Before feature extraction, we spatially filter the signals from the six electrodes using Common Average Referencing (CAR) [2] method to reduce the effect of neighboring locations from these signals. Then, we temporally band pass filter the signals in the bandwidth of $8-25 \mathrm{~Hz}$ using an IIR elliptical filter of order 6, pass-band attenuation of $50 \mathrm{~dB}$ and stop-band attenuation of $0.5 \mathrm{~dB}$. The merit of selecting elliptical filter lies in its good frequency-domain characteristics of sharp roll-off, and independent control over the pass-band and stop-band ripples [15].

Based on the timing sequence of the visual cue, sample points from the 2nd second to the 5th second ( 3 seconds in total) are extracted from each trial for data analysis.

\section{$2.4 \quad$ Feature Extraction}

Following pre-processing, we apply Welchs' based power spectral density to determine the feature vectors of the EEG signals at each trial. Welchs' method is a parametric method of estimation of power spectral density [16]. Power spectral density (PSD) portrays the distribution of power in the frequency domain. Welch method divides the time series data, $x(n)$ into possibly overlapping segments over a length $L$, a weighting vector $w_{k}$ is applied to each segment and a modified periodogram of each segment is computed using a discrete Fourier transform(DFT) thereby averaging the PSD estimates. Thus Welch PSD estimate can thus be summed up as,

$$
P\left(w_{k}\right)=\sum_{n=0}^{N-1}\left|D F T\left(x_{n}\right)\right|^{2}
$$

In this paper we have selected a hamming window of size 250 over the complete frequency range and an overlap percentage of $50 \%$. From the whole frequency range of 0 to $125 \mathrm{~Hz}$ (since $250 \mathrm{~Hz}$ is the sampling frequency), only 
estimates from the bands of $8-12 \mathrm{~Hz}$ and $16-24 \mathrm{~Hz}$ are selected to construct the feature vector. The final dimension of the feature vector is 14. Fig. 3 illustrates an example of the power spectral density estimates of fast and slow movement for both limbs based on the EEG obtained from channel location C3.

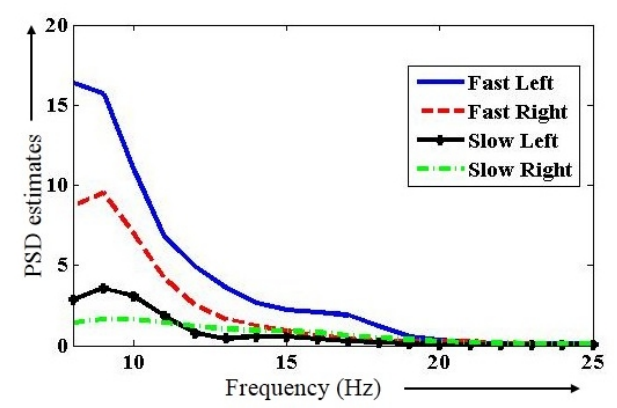

Fig. 3. The power spectral density estimates from electrode C3 for the four different mental tasks performed by a subject.

\subsection{Classification}

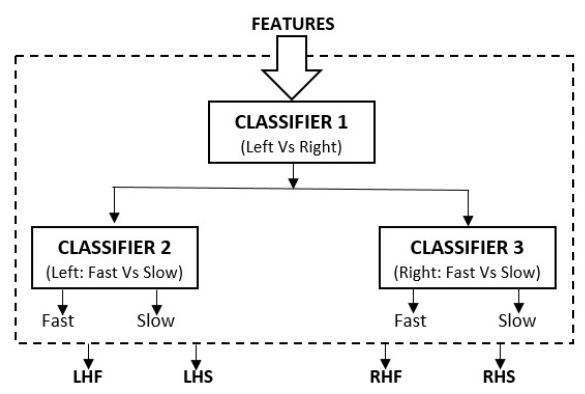

Fig. 4. The hierarchical classification strategy implemented in this study.

The classification scheme implemented in this study is shown in Fig. 4. Two levels of hierarchical classifiers are implemented, where the first level classifies between the left and right hand movement (Classifier 1) and the second level differentiates between fast and slow movement (Classifier 2 and 3). Consequently, the final output is in the form of right-hand fast movement (RHF), left-hand fast movement (LHF), right-hand slow movement (RHS) and left-hand slow movement (LHS). 
In this paper, we have used support vector machine (SVM), naïve Bayesian $(\mathrm{NB})$, linear discriminant analysis (LDA) and k-nearest neighbor $(\mathrm{kNN})$ to distinguish between the different levels of classifiers [9].

\section{$3 \quad$ Results and Discussions}

The analysis of the whole experiment is performed in a Matlab 2012b platform on a computer with the following specifications: Processor Intel i7 @ $3.2 \mathrm{GHz}, 8$ GB RAM.

The total feature vector is partitioned into two separate datasets, training and testing dataset, over 10 runs using $\mathrm{k}$-fold cross validation approach [17]. Classification Accuracy and Computational Time are the metrics used to analyze the dataset using the four classifiers: SVM, NB, LDA, and kNN and the results are shown in Table 1. As noted in the table, all the three classifiers: Classifier 1 (CL1), Classifier 2 (CL2), and Classifier 3 (CL3), yields an accuracy of more than $75 \%$. Also, the three NB classifiers produces the highest accuracy (more than $95 \%$ ) as compared to the other classifiers. As observed from Table 1, NB classifier maintains a fine trade-off between the accuracies and the computational time taken to process the result.

Table 1. Accuracies of the classifiers obtained during training for 9 subjects

\begin{tabular}{|c|c|c|c|c|}
\hline S.ID & SVM & NB & LDA & $\mathrm{kNN}$ \\
\hline & \multicolumn{4}{|c|}{ CL1 CL2 CL3 CL1 CL2 CL3 CL1 CL2 CL3 CL1 CL2 CL3 } \\
\hline 1 & 88.282 .376 .4100 & 10094.1 & .382 .376 .4 & 88.282 .376 .4 \\
\hline 2 & 76.476 .476 .4100 & 10094.1 & 82.382 .376 .4 & 88.28 \\
\hline 3 & 88.270 .570 .5100 & 10094.1 & 82.376 .470 .5 & 88.276 .476 .4 \\
\hline 4 & $82.382 .3 \quad 76.494 .1$ & 100100 & 88.276 .476 .4 & 82.382 .376 .4 \\
\hline 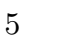 & 76.470 .570 .5100 & 94.1100 & 82.376 .47 & 82.38 \\
\hline 6 & $\begin{array}{llll}88.2 & 70.5 & 70.5 & 100\end{array}$ & 10094.1 & 88.288 .276 .4 & 88.282 .382 .3 \\
\hline 7 & \multirow{2}{*}{$\begin{array}{llll}82.3 & 88.2 & 82.3 & 94.1 \\
76.4 & 82.3 & 82.3 & 94.1\end{array}$} & 94.1100 & $82.388 .2 \quad 82.3$ & 76.476 \\
\hline 8 & & 94.194 .1 & 76.482 .382 .3 & 76.482 .388 .2 \\
\hline 9 & 82.376 .476 .494 .1 & 94.1100 & 76.482 .382 .3 & 82.382 .382 .3 \\
\hline \multicolumn{5}{|c|}{ Avg. 82.377 .775 .897 .397 .396 .782 .381 .777 .183 .681 .781 .0} \\
\hline & 0.3107 & 0.0071 & 0.0330 & 0.0338 \\
\hline
\end{tabular}

We have also employed Friedman Test [18] to statistically validate our results. The significance level is set at $\alpha=0.05$. The null hypothesis here, states that all the algorithms are equivalent, so their ranks should be equal. We consider the mean classification accuracy (from Table 2) as the basis of ranking. Table 2 provides the ranking of each classifier algorithm.

Now, from Table 2, the $\chi_{F}^{2}$ for the three classifiers $(C L 1, C L 2, C L 3)=$ $(16.44,17.76,20.61)>=9.488$, So, the null hypothesis, claiming that all the 
Table 2. Accuracies of the classifiers obtained during training for 9 subjects

\begin{tabular}{|c|c|c|c|c|c|c|c|c|c|c|c|c|}
\hline \multirow[t]{2}{*}{ S.ID } & \multicolumn{3}{|c|}{ SVM } & \multicolumn{3}{|c|}{ NB } & \multicolumn{3}{|c|}{ LDA } & \multicolumn{3}{|c|}{$\mathrm{kNN}$} \\
\hline & CL1 & & & & & & CL1 & $C_{1}$ & CL3 & CL1 & & CL3 \\
\hline 1 & 2.5 & 3 & 2 & 1 & 1 & & 4 & 3 & 3 & 2.5 & 3 & 3 \\
\hline 2 & 4 & 1 & 3. & 1 & 1 & & 3 & 3 & 3.5 & 2 & 2 & \\
\hline 3 & 2.5 & 4 & 3.5 & 1 & 1 & $I$ & 4 & 2.5 & 3.5 & 2.5 & 2.5 & 2 \\
\hline 4 & 3.5 & 2.5 & 3 & 1 & 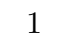 & & 2 & 4 & 3 & 3.5 & 2.5 & 3 \\
\hline 5 & 4 & 4 & 3.5 & 1 & 1 & & 2.5 & 2.5 & 3.5 & 2.5 & 2.5 & 2 \\
\hline 6 & 3 & 4 & 4 & 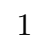 & 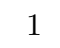 & & 3 & 2 & 3 & 3 & 3 & 2 \\
\hline 7 & 2.5 & 2.5 & 3.5 & 1 & 1 & 1 & 2.5 & 2.5 & 3.5 & 4 & 4 & 2 \\
\hline 8 & 3 & 3 & 3.5 & 1 & 1 & 1 & 3 & 3 & 3.5 & 3 & 3 & 2 \\
\hline 9 & 2.5 & 4 & 4 & 1 & 1 & 1 & 4 & 2.5 & 2.5 & 2.5 & 2.5 & 2.5 \\
\hline $\mathbf{R j}$ & & 4 & 3.5 & 1 & 1 & 1 & & & & & & \\
\hline
\end{tabular}

algorithms are equivalent, is wrong and, therefore, the performances of the algorithms are determined by their ranks only. It is clear from the table that the rank of NB is 1, claiming NB outperforms all the algorithms by Friedman Test.

\section{Conclusion and Future Direction}

The paper proposes a classification strategy towards discriminating between fast and slow movement of the left and right hand. The feature vectors are prepared using a power spectral density estimates using Welch method and are fed as inputs to the SVM, NB, LDA and kNN classifiers. Here, we have incorporated two levels of classification: the first level classifies between left and right movement and the second level classifies between fast and slow movement. The results show that NB classifier yields the best results with $97.1 \%$ of average classification accuracy in 0.0541 seconds.

Based on the promising result obtained in this study, we propose to move forward towards real-time control problems in our future study. We intend to control the movement of a robotic arm which can further help in the development of neuro-prosthetics in future.

Acknowledgments. I would like to thank University Grants Commission, India, University of Potential Excellence Programme (Phase II) in Cognitive Science, Jadavpur University and Council of Scientific and Industrial Research, India.

\section{References}

1. Daly, J.J., Wolpaw, J.R.: Brain-computer interfaces in neurological rehabilitation. Lancet Neurol 7, 10321043 (2008) 
2. Dornhege, G.: Towards Brain-Computer Interfacing. MIT Press (2007)

3. McFarland, D.J., Wolpaw, J.R.: Brain-computer interface operation of robotic and prosthetic devices. Computer 41(10), 52-56 (2008)

4. Bermudez i Badia, S., Garcia Morgade, A., Samaha, H., Verschure, P.F.M.J.: Using a Hy-brid Brain Computer Interface and Virtual Reality System to Monitor and Promote Cortical Reorganization through Motor Activity and Motor Imagery Training. IEEE Trans. Neural Sys. Rehab. Eng. 21 (2): 174-181 (2013)

5. Bordoloi , S., Sharmah, U., Hazarika, S.M.: Motor imagery based BCI for a maze game. In: 4th Int. Conf. Intelligent Human Computer Interaction (IHCI),Kharagpur, India ,pp. 1-6 (2012)

6. Millan, J.R., Rupp, R., Muller-Putz, G.R., Murray-Smith, R., Giugliemma, C., Tangermann, M., Vidaurre, C., Cincotti, F., Kubler, A., Leeb, R., Neuper, C., Muller, K.R., Mattia, D.: Combining brain-computer interfaces and assistive technogies: State-of-the-art and challenges. Front. Neurosci. 4: 1-15 (2010)

7. Bhattacharyya, S., Sengupta, A., Chakraborti, T., Konar, A., Tibarewala, D.N.: Automatic feature selection of motor imagery EEG signals using differential evolution and learning automata. Med. \& Bio. Eng. \& Comp. 52(2), 131-139 (2014)

8. Zhou, W., Zhong, L., Zhao, H.: Feature Attraction and Classification of Mental EEG Using Approximate Entropy, In: 27th Ann. Int. Conf. Eng. Med. \& Bio. Soc., $59755978(2005)$

9. Theodoridis, S., Koutroumbas, K.: Pattern Recognition, 4th Edition. Academic Press, pp 13-322 (2009)

10. Qiang, C., Hu, P., Huanqing, F.: Experiment study of the relation between motion complexity and event-related desynchronization/synchronization. In: 1st Int. Conf. Neural Interface \& Cont. 2005, 14-16 (2005)

11. Chai, R., Ling, S.H., Hunter, G.P., Nguyen, H.T.: Mental non-motor imagery tasks classifi-cations of brain computer interface for wheelchair commands using genetic algorithm-based neural network. In: The 2012 Int. Joint Conf. Neural Networks, 1-7 (2012)

12. Bhattacharyya, S., Khasnobish, A., Chatterjee, S., Konar, A., Tibarewala, D.N.: Performance analysis of LDA, QDA and KNN algorithms in left-right limb movement classification from EEG data. In: 2010 Int. Conf. Sys. Med. \& Bio., 126-131 (2010)

13. Khasnobish, A., Bhattacharyya, S., Konar, A., Tibarewala, D.N., Nagar, A.K.: A two-fold classification for composite decision about localized arm movement from EEG by SVM and QDA techniques, In: The 2011 Int. Joint Conf. Neural Networks, 1344-1351 (2011)

14. Pfurtscheller, G., Neuper, C., Schl, A., \& Lugger, K.: Separability of EEG Signals Recorded During Right and Left Motor Imagery Using Adaptive Autoregressive Parameters, IEEE Trans. Rehab. Engg. 6(3), 316325 (1998)

15. Palaniappan, R.: Brain computer interface design using band powers extracted during mental tasks. In: Conference Proceedings of 2nd International IEEE EMBS Conference on Neural Engineering, 321-324 (2005)

16. D.G. Childers, Modern spectrum analysis, IEEE Press, 1978.

17. Kohavi, R.: A study of cross-validation and bootstrap for accuracy estimation and model selection. In: Int. Joint Conf. on Artificial Intelligence, 14(2), 1137-1145 (1995)

18. Theodorsson-Norheim, E.: Friedman and Quade tests: BASIC computer program to perform non-parametric two-way analysis of variance and multiple comparisons on ranks of several related samples. Comp. Bio. \& Med. 17(2), 85-99 (1987) 